\title{
Metric Free Nearness Measure using Description-based Neighbourhoods
}

\author{
Christopher J. Henry
}

\begin{abstract}
The focus of this paper is on a metric free nearness measure for quantifying the descriptive nearness of digital images. Regions Of Interest (ROI) play an important role in discerning perceptual similarity within a single image, or between a pair of images. In terms of pixels, closeness between ROIs can be assessed in light of the traditional closeness between points and sets and closeness between sets using topology or proximity theory. A metric free nearness measure is introduced in this paper by finding common patterns among disjoint description based neighbourhoods obtained from these spatially defined sets. The contribution of this article is a metric free nearness measure implemented within the Proximity System, an application used to demonstrate near set concepts using digital images.

MSC2010 Classification: Primary: 54E05 (Proximity structures and generalizations), 62H35 (image analysis), Secondary: 68U10 (image processing), 68N01 (software).
\end{abstract}

Keywords. Nearness measure, digital image, near sets, region of interest, description based neighbourhood.

\section{Introduction}

The inspiration for the approach presented in this article is an observation in [1] that the concept of nearness* is a generalization of set intersection. The idea follows from the notion of set description $[3, \S 4.3]$, which is a collection of the unique feature vectors ( $n$-dimensional real-valued feature vectors representing characteristics of the objects) associated with all the

Springer Mathematics in Computer Science Special Issue.

*Introduced within the context of Riesz's proximity [2]. 
objects in the set. Describing sets in this manner, at some level, matches the human approach to describing sets of objects. Furthermore, in comparing disjoint sets of objects, we must at some level be performing a comparison of the descriptions we associate with the objects within the sets. Thus, a natural approach for quantifying the degree of similarity (i.e. the nearness or apartness) between two sets would be to look at the intersection of the sets containing their unique feature vectors.

The sets considered in this article are obtained from digital images. Specifically, Regions Of Interest (ROI) play an important role in discerning perceptual similarity within a single image, or between a pair of images. In this work, four different ROIs are considered. Namely, a simple set of pixels, a spatial neighbourhood, a descriptive neighbourhood, and a hybrid approach in which the neighbourhood is formed by spatial and descriptive characteristics of the objects. In terms of pixels, closeness between ROIs can be assessed in light of the traditional closeness between points and sets and closeness between sets using topology or proximity theory $[3,4]$.

The approach reported here builds on the work of many others. The idea of sets of similar sensations was first introduced by J. H. Poincaré in which he reflects on experiments performed by E. Weber in 1834, and G. T. Fechner's insight in 1850 [5, 6, 7, 8]. Poincaré's work was inspired by Fechner, but the key difference is Poincaré's work marked a shift from stimuli and sensations to an abstraction in terms of sets together with an implicit idea of tolerance. Next, the idea of tolerance is formally introduced by E. C. Zeeman [9] with respect to the brain and visual perception. Zeeman makes the observation that a single eye cannot identify a 2D Euclidean space because the Euclidean plane has an infinite number of points. Instead, we see things only within a certain tolerance. This idea of tolerance is important in mathematical applications where systems deal with approximate input and results are accepted with a tolerable level of error, an observation made by A. B. Sossinsky [5], who also connected Zeeman's work with that of Poincaré's. In addition to these ideas on tolerance, F. Riesz first published a paper in 1908 on the nearness of two sets $[2,10]$, initiating the mathematical study of proximity spaces and the eventual discovery of descriptively near sets. Specifically, Near set theory was inspired by a collaboration in 2002 by Z. Pawlak and J. F. Peters on a poem entitled "How Near" [11], which lead to the introduction of descriptively near sets $[12,13]$. Next, tolerance near sets were also introduced by Peters $[14,15]$, which combines near set theory with the ideas of tolerance spaces and relations. Finally, a tolerance-based nearness measure was introduced in [16, 17]. 
The contributions of this article are several new description-based operators and some of their properties, and a new metric-free description-based nearness measure. The outline of this article is as follows. Section 2 briefly describes the Proximity System, the system used to generate the examples give in this article. Next, Section 3 defines some description-based set operators and examines some traditional set properties on these new operators. Section 4 outlines several types of neighbourhoods to which the descriptive-based operators can be applied. Section 5 defines the new metric-free nearness measure.

\section{Proximity System}

The examples presented in this article were generated with the Proximity System (shown in Fig. 1), an application being developed to demonstrate the descriptive-based approaches introduced in this article within the context of digital image analysis. The Proximity System was written in Java and is intended to run in two different operating environments, namely on Android 4.0 enabled smartphones and tablets, as well as any operating system running a Java Virtual Machine. Specifically, both applications uses the same back-end libraries to perform the description-based calculations, where the only differences are the user interface and the Android 4.0 version has less available features (i.e. probe functions given in Definition 3) due to restrictions on system resources. A detailed survey of the system is outside the scope of this paper, however, the system is freely available for download ${ }^{\dagger}$. Finally, to facilitate the introduction of examples in Section 3, the digital images processed by the Proximity System are formally given as follows. Define an RGB image as $f=\left\{\mathbf{p}_{1}, \mathbf{p}_{2}, \ldots, \mathbf{p}_{N}\right\}$, where $\mathbf{p}_{i}=\left(c_{i}, r_{i}, R_{i}, G_{i}, B_{i}\right)^{\mathrm{T}}$, $c_{i} \in[1, W], r_{i} \in[1, H], R_{i}, G_{i}, B_{i} \in[0,255]$, and $W, H$ respectively denote the width and height of the image and $W \times H=N$. Moreover, within the context of the Proximity System, define $O$ as the set of all pixels, i.e. $O=f=\left\{\mathbf{p}_{1}, \mathbf{p}_{2}, \ldots, \mathbf{p}_{N}\right\}$.

\section{Description-based Set Operators}

Many interesting properties can be considered by introducing the description of a set. The following subsections give definitions and properties of new operators considered in the light of object descriptions.

\footnotetext{
${ }^{\dagger}$ As is the case with the NEAR System [18] available at http://wren.ee.umanitoba.ca/
} 


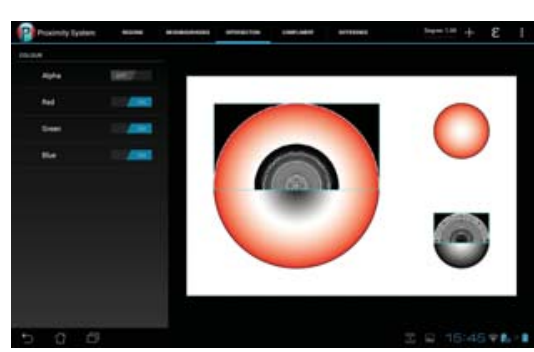

(a)

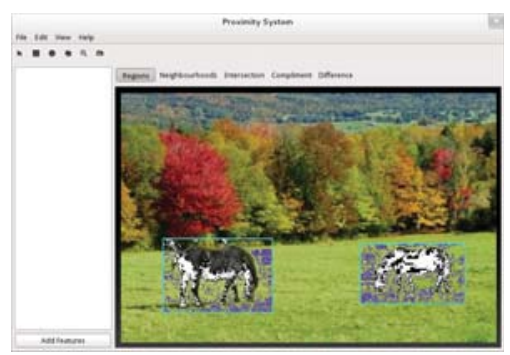

(b)

Figure 1. Screenshots of Proximity System prototype: a) Android 4 application, and b) Desktop application.

\subsection{Definitions}

A logical starting point for introducing descriptive-based operators begins with establishing a basis for describing elements of sets. All sets in this work consist of perceptual objects.

Definition 1. Perceptual Object. A perceptual object is something that has its origin in the physical world.

A perceptual object is anything in the physical world with characteristics observable to the senses such that they can be measured and are knowable to the mind. In keeping with the approach to pattern recognition suggested by M. Pavel [19], the features of a perceptual object are quantified by probe functions.

Definition 2. Feature [20]. A feature characterizes some aspect of the makeup of a perceptual object.

Definition 3. Probe Function [12,21]. A probe function is a real-valued function representing a feature of a perceptual object.

Next, a perceptual system is a set of perceptual objects, together with a set of probe functions.

Definition 4. Perceptual System [22]. A perceptual system $\langle O, \mathbb{F}\rangle$ consists of a non-empty set $O$ of sample perceptual objects and a non-empty set $\mathbb{F}$ of real-valued functions $\phi \in \mathbb{F}$ such that $\phi: O \rightarrow \mathbb{R}$.

Combining Definitions $1 \& 3$, the description of a perceptual object within a perceptual system can be defined as follows. 
Definition 5. Object Description. Let $\langle O, \mathbb{F}\rangle$ be a perceptual system, and let $\mathcal{B} \subseteq \mathbb{F}$ be a set of probe functions. Then, the description of a perceptual object $x \in O$ is a feature vector given by

$$
\Phi_{\mathcal{B}}(x)=\left(\phi_{1}(x), \phi_{2}(x), \ldots, \phi_{i}(x), \ldots, \phi_{l}(x)\right),
$$

where $l$ is the length of the vector $\Phi_{\mathcal{B}}$, and each $\phi_{i}(x)$ in $\Phi_{\mathcal{B}}(x)$ is a probe function value that is part of the description of the object $x \in O$.

Note, the idea of a feature space is implicitly introduced along with the definition of object description. An object description is the same as a feature vector as described in traditional pattern classification [23]. The description of an object can be considered a point in an $l$ dimensional Euclidean space $\mathbb{R}^{l}$ called a feature space. Further, a collection of these points, i.e., a set of objects $A \subseteq O$, is characterized by the unique description of each object in the set.

Definition 6. Set Description [3, $§ 4.3]$. Let $A$ be a set. Then the set description of $A$ is defined as

$$
\Phi(A)=\{\Phi(a): a \in A\}
$$

Example 1. Let $\langle O, \mathbb{F}\rangle$ be a perceptual system, where $O$ contains the pixels in Fig. $2, A \subseteq O$, and $\mathcal{B} \subseteq \mathbb{F}$ contains probe functions based on the RGB colour model. Then, the set description of $A$ is $\Phi(A)=\{\square, \square, \square, \square, \square\}$, where each coloured box represents the 3 -dimensional real-valed rgb vector associated the box's colour.

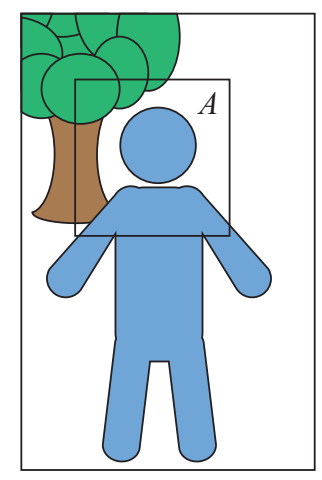

Figure 2. Example demonstrating Definition 6.

Lemma 1. The set description of the empty set is the empty set. 
Proof. Obvious.

Next, J. Peters and S. Naimpally observed that, from a spatial point of view, the idea of nearness is a generalization of set intersection [1]. In other words, when considering the metric proximity, two sets are near each other when their intersection is not the empty set. Furthermore, they applied this idea to the concept of descriptive nearness in $[3, \S 4.3]$ by focusing on the descriptions of objects within the sets. In this case, two sets are considered near each other if the intersection of their descriptions is not the empty set. The following definitions build on these concepts and provide the foundation for demonstrating the properties of Table 1 within a description-based nearness framework.

Definition 7. Descriptive Set Union. Let $A$ and $B$ be any two sets. The descriptive (set) union of $A$ and $B$ is defined as

$$
A \underset{\Phi}{\cup} B=\{x \in A \cup B: \Phi(x) \in \Phi(A) \text { or } \Phi(x) \in \Phi(B)\}
$$

Theorem 1. The descriptive union is equivalent to set union.

$$
A \cup \underset{\Phi}{\cup} B \Longleftrightarrow A \cup B
$$

Proof. Let $a \in A$ and $b \in B$. Since, $\Phi(a) \in \Phi(A) \forall a \in A$ (by Definition 6), $A \subseteq A \cup B$.

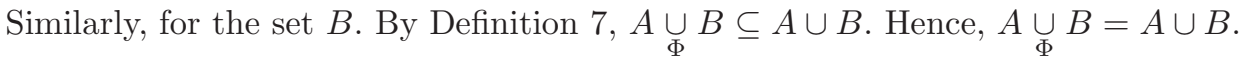

As a result of Theorem 1, only counter examples to the properties involving set union in Table 1 will be considered in this article.

Definition 8. Descriptive Set Intersection $[1,3]$. Let $A$ and $B$ be any two sets. The descriptive (set) intersection of $A$ and $B$ is defined as

$$
A \underset{\Phi}{\cap} B=\{x \in A \cup B: \Phi(x) \in \Phi(A) \text { and } \Phi(x) \in \Phi(B)\} .
$$

Example 2. Let $\left\langle O_{1}, \mathbb{F}\right\rangle$ and $\left\langle O_{2}, \mathbb{F}\right\rangle$ be perceptual systems corresponding to Fig. 3a \& 3c, respectively, where the perceptual objects and probe functions are defined in the same manner as Example 1. Moreover, let the blue rectangles in Fig. 3b (resp. Fig. 3d) represent two sets, $A, B$, for which the descriptive intersection is considered. Then, the inverted pixels (i.e. $p_{i}=$ $\left.\left(c_{i}, r_{i}, 255-R_{i}, 255-G_{i}, 255-B_{i}\right)^{\mathrm{T}}\right)$ within these sets represent their descriptive intersection, i.e. the inverted pixels represent the objects with matching descriptions in both sets. 


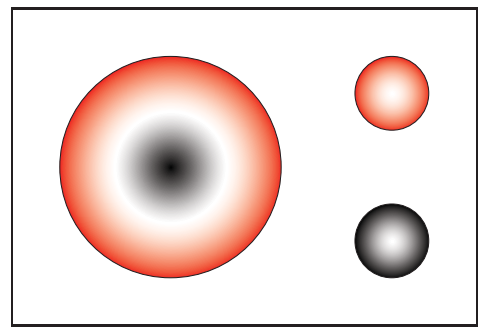

(a)

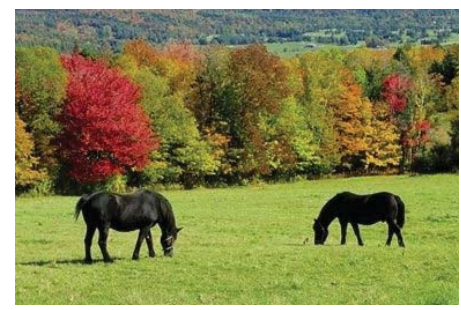

(c)

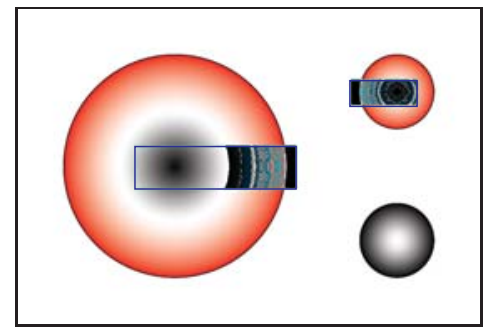

(b)

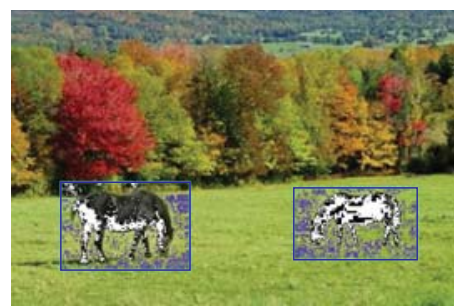

(d)

Figure 3. Example demonstrating Definition 8.

Definition 9. Descriptive Set Difference. The descriptive (set) difference (or descriptive difference set) between two sets $A$ and $B$ is defined as

$$
A \backslash_{\Phi} B=\{x \in A: \Phi(x) \notin \Phi(B)\} .
$$

Example 3. The descriptive difference between the sets introduced in Example 2 are given Fig. 4. In this case, the inverted pixels represent all the objects that do not have matching descriptions in the other set.

Lemma 2. The descriptive difference of a set with itself is the empty set.

$$
A \backslash_{\Phi} A=\emptyset
$$

Proof.

$$
\begin{aligned}
A \backslash_{\Phi} A & \Longleftrightarrow x \in A: \Phi(x) \notin \Phi(A), \quad \text { (by Definition 9) } \\
& \Longleftrightarrow \emptyset .
\end{aligned}
$$




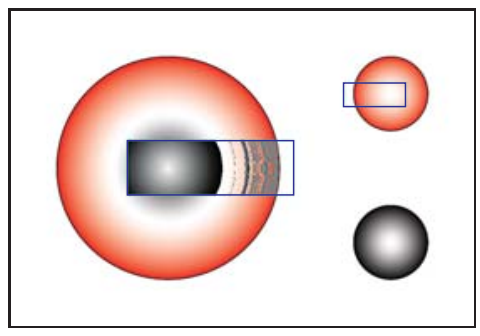

(a)

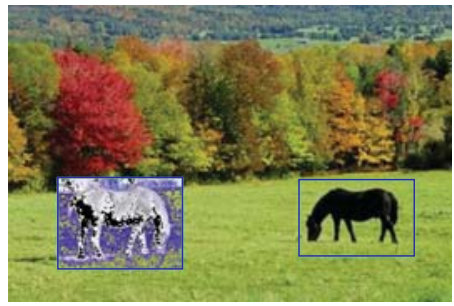

(b)

Figure 4. Example demonstrating Definition 9.

Definition 10. Relative Descriptive Complement. Let $A$ be a set, and let $B \subseteq A$. Then, the descriptive difference can also be called the relative descriptive complement of $B$ in $A$, and is defined as

$$
\underset{\Phi}{\complement_{A}}(B)=A \underset{\Phi}{\backslash} B=\{x \in A: \Phi(x) \notin \Phi(B)\}
$$

Lemma 3. The relative descriptive complement of a set in itself is the empty set.

$$
\underset{\Phi}{\complement_{A}}(A)=\emptyset
$$

Proof.

$$
\begin{aligned}
\complement_{A}(A) & \Longleftrightarrow x \in A: \Phi(x) \notin \Phi(A), \text { (by Definition 10) } \\
& \Longleftrightarrow \emptyset .
\end{aligned}
$$

Lemma 4. The relative descriptive complement of the empty set is the set itself.

$$
\underset{\Phi}{\complement_{A}}(\emptyset)=A
$$

Proof.

$$
\begin{aligned}
\underset{\Phi}{\complement_{A}(\emptyset)} & \Longleftrightarrow x \in A: \Phi(x) \notin \Phi(\emptyset), \text { (by Definition } 10 \text { and Lemma } 1 \text { ) } \\
& \Longleftrightarrow A .
\end{aligned}
$$


Definition 11. Descriptive Set Complement. The descriptive (set) complement of a set $A$ in the universe $U$ is defined as

$$
\underset{\Phi}{\complement}(A)=\underset{\Phi}{\complement_{U}}(A)=U \backslash_{\Phi} A
$$

Example 4. Considering the perceptual systems introduced in Example 2, the descriptive complement of each set represented by a blue rectangles in Fig. 5 is given by the inverted pixels. In other words, the inverted pixels represent objects that do not have matching descriptions to those contained inside the blue rectangle.

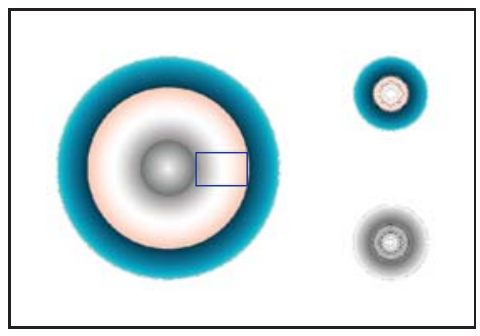

(a)

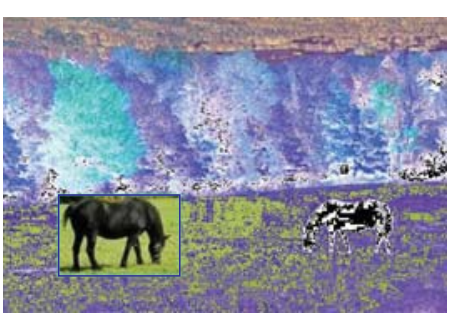

(b)

Figure 5. Example demonstrating Definition 11.

Lemma 5. The descriptive complement is not the same as the set complement.

$$
\complement_{\Phi}(A) \neq \complement(A)
$$

Proof. Let $x \in U \backslash A$, and $\Phi(x) \in \Phi(A)$ (i.e. $x$ has the same description as an object in $A$ ). By Definition 11, $x \notin \underset{\Phi}{\complement}(A)$, and, consequently, $\underset{\Phi}{\complement}(A) \neq \complement(A)$.

\subsection{Properties}

This section presents properties of the definitions introduced in Section 3.1, where the choice of properties highlighted here was guided by the list in $[24, \S \mathrm{R} 1.14]$ and are summarized in Table 1.

Theorem 2. The descriptive complement of the universe is the empty set.

$$
\underset{\Phi}{\complement}(U)=\emptyset
$$

Proof. Substitute $U$ for $A$ in $\underset{\Phi}{\complement_{A}}(A)$ (see Lemma 3 ). 
TABle 1. Tolerance Class Example

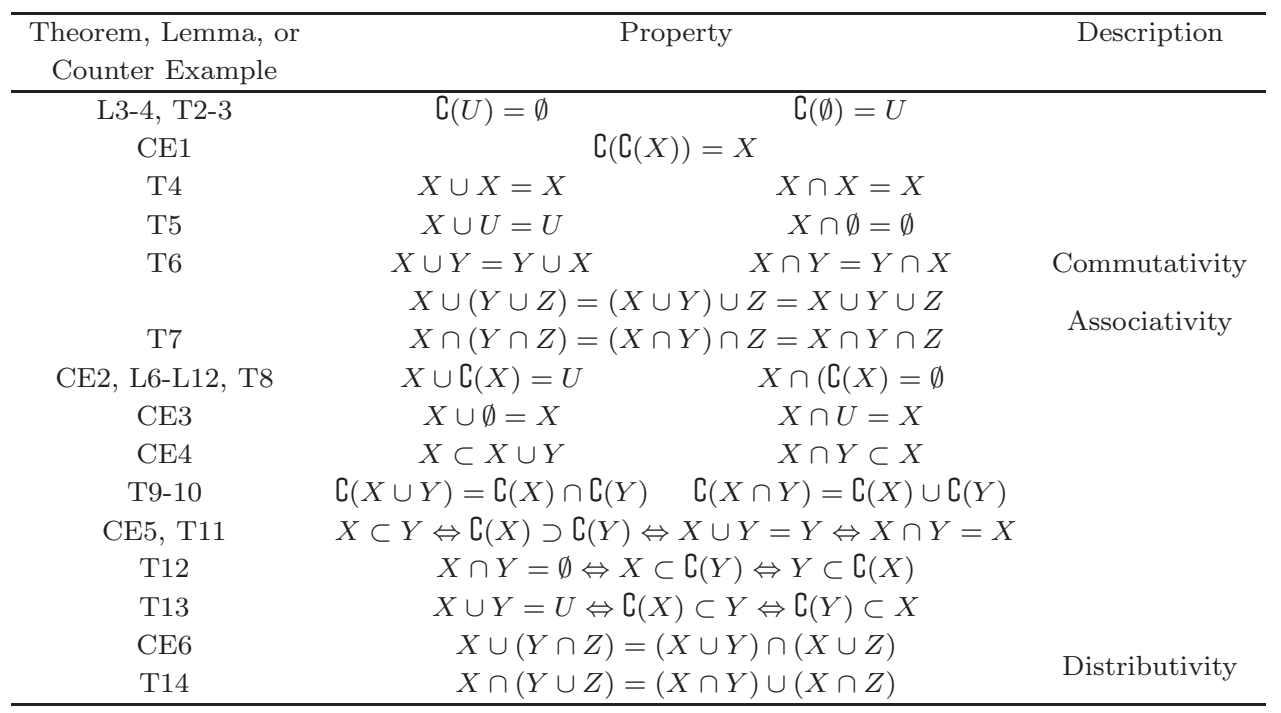

Theorem 3. The descriptive complement of the empty set is the universe.

$$
\underset{\Phi}{\complement}(\emptyset)=U
$$

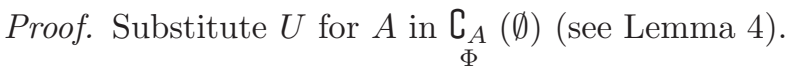

Counter Example 1. The descriptive complement of the descriptive complement of a set is not the original set.

$$
\underset{\Phi}{\left.\complement_{\Phi}(A)\right) \neq A}
$$

Proof. By Definition 11, $\underset{\Phi}{\complement}(A)=\{x \in U: \Phi(x) \notin \Phi(A)\}$. Let $x \in U \backslash A, a \in A$, and $\Phi(x)=$ $\Phi(a)$. Then, $\underset{\Phi}{\complement}(A) \neq U \backslash A$. Consequently, $\underset{\Phi}{\complement} \underset{\Phi}{\complement}(A))$ will contain objects from $U \backslash A$ (as shown in Fig. 6).

Theorem 4. The descriptive intersection is idempotent.

$$
A \bigcap_{\Phi} A=A
$$




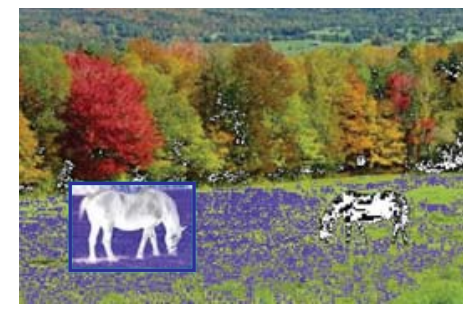

Figure 6. Instance of Counter Example 1.

Proof.

$$
\begin{aligned}
A \bigcap_{\Phi} A & \Longleftrightarrow x \in A \cup A: \Phi(x) \in \Phi(A) \text { and } \Phi(x) \in \Phi(A), \text { (by Definition 8) } \\
& \Longleftrightarrow x \in A: \Phi(x) \in \Phi(A), \text { (since set union is idempotent) } \\
& \Longleftrightarrow A \text { (by Definition } 6) .
\end{aligned}
$$

Counter Example 2. The descriptive union of a set and its descriptive complement is not the universe.

$$
A \underset{\Phi}{\cup} \underset{\Phi}{\complement}(A) \neq U
$$

Proof. Similar to Counter Example 1

Theorem 5. The descriptive intersection of any set with the empty set is itself the empty set.

$$
A \underset{\Phi}{\cap} \emptyset=\emptyset
$$

Proof.

$$
\begin{aligned}
A \cap \emptyset & \Longleftrightarrow x \in A \cup \emptyset: \Phi(x) \in \Phi(A) \text { and } \Phi(x) \in \Phi(\emptyset), \text { (by Definition } 8) \\
& \Longleftrightarrow x \in A: \Phi(x) \in \Phi(A) \text { and } \Phi(x) \in \emptyset, \quad \text { (by Lemma 1) } \\
& \Longleftrightarrow \emptyset .
\end{aligned}
$$

Theorem 6. Descriptive intersection is commutative.

$$
A \bigcap_{\Phi} B=B \bigcap_{\Phi} A
$$


Proof.

$$
\begin{aligned}
x \in A \underset{\Phi}{\cap} B & \Longleftrightarrow x \in A \cup B: \Phi(x) \in \Phi(A) \text { and } \Phi(x) \in \Phi(B), \text { (by Definition 8) } \\
& \Longleftrightarrow x \in A \cup B: \Phi(x) \in \Phi(B) \text { and } \Phi(x) \in \Phi(A), \\
& \Longleftrightarrow B \underset{\Phi}{\cap} A .
\end{aligned}
$$

Theorem 7. Descriptive intersection is associative.

$$
A \underset{\Phi}{\bigcap}(B \underset{\Phi}{\bigcap} C)=(A \underset{\Phi}{\bigcap} B) \underset{\Phi}{\bigcap} C
$$

Proof.

$$
\begin{aligned}
& x \in A \underset{\Phi}{\cap}(B \underset{\Phi}{\bigcap} C) \\
\Longleftrightarrow & x \in A \cup B \cup C: \Phi(x) \in \Phi(A) \text { and }(\Phi(x) \in \Phi(B) \text { and } \Phi(x) \in \Phi(C)), \text { (by Definition 8) } \\
\Longleftrightarrow & x \in A \cup B \cup C:(\Phi(x) \in \Phi(A) \text { and } \Phi(x) \in \Phi(B)) \text { and } \Phi(x) \in \Phi(C), \\
\Longleftrightarrow & x \in\left(A \bigcap_{\Phi} B\right) \bigcap_{\Phi} C .
\end{aligned}
$$

Next, Lemmas 6-12 are introduced to prove the descriptive intersection of a set and its descriptive complement is the empty set (given in Theorem 8).

Lemma 6. Let $A, B \subseteq C$. Then, the descriptive difference between $A$ and $B$ is a subset of the descriptive intersection with the relative descriptive complement with respect to $C$.

$$
A \backslash B \subseteq A \underset{\Phi}{\cap} \underset{\Phi}{\complement_{C}}(B)
$$

Proof.

$$
\begin{aligned}
A \backslash_{\Phi} B & =\{x \in A: \Phi(x) \notin \Phi(B)\}, \text { (by Definition 9) } \\
& \subseteq\left\{x \in A: \Phi(x) \in \complement_{\Phi}(B)\right\}, \text { (by Definition 10) } \\
& \subseteq A \underset{\Phi}{\cap} \underset{\Phi}{\complement_{C}}(B) . \text { (by Definition 8) }
\end{aligned}
$$


Note, for Lemma 6, equivalence cannot be guaranteed, which is demonstrated in the following example.

Example 5. Let $\langle C, \mathbb{F}\rangle$ be a perceptual system, where $C$ contains the pixels in Fig. 7, $A, B \subseteq C$, and $\mathcal{B} \subseteq \mathbb{F}$ contains probe functions based on the RGB colour model. Then, $A \backslash \underset{\Phi}{\backslash} B=\left\{x_{1}\right\}$, $\underset{\Phi}{\complement_{C}}(B)=\left\{x_{1}, x_{3}, x_{4}, x_{5}\right\}$, and $\underset{\Phi}{A} \underset{\Phi}{\cap} \complement_{C}(B)=\left\{x_{1}, x_{3}, x_{4}, x_{5}\right\}$.

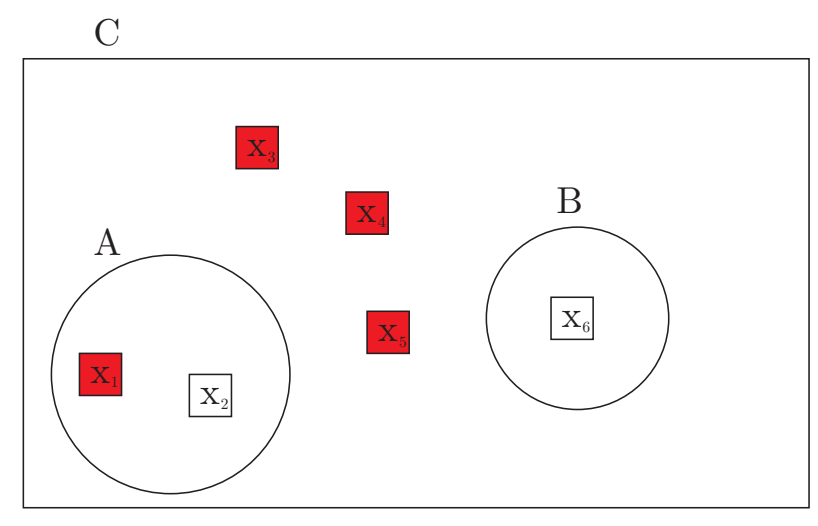

FiguRE 7. Example demonstrating Lemma 6.

Lemma 7. The descriptive difference is a subset of the descriptive intersection with the descriptive complement.

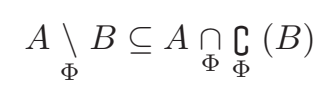

Proof. This follows directly from Lemma 6 . Here, let $C=U$. Since, $A, B \subseteq U$, the result follows.

Lemma 8. Let $A, B, C$ be sets. Then,

$$
\left(A \bigcap_{\Phi} B\right) \underset{\Phi}{\backslash} C \subseteq\left(A \backslash \underset{\Phi}{\backslash}{\underset{\Phi}{\bigcap}}_{(B \backslash} C\right) .
$$


Proof.

$$
\begin{aligned}
& (A \underset{\Phi}{\cap} B) \underset{\Phi}{\backslash} C \subseteq(A \underset{\Phi}{\cap} B) \underset{\Phi}{\cap} \underset{\Phi}{\complement}(C), \text { (by Lemma 7) } \\
& \subseteq\left(A \bigcap_{\Phi} B\right) \bigcap_{\Phi}\left(\underset{\Phi}{\complement}(C) \underset{\Phi}{\bigcap_{\Phi}}(C)\right), \text { (by Theorem 4) } \\
& \subseteq(A \underset{\Phi}{\bigcap} \underset{\Phi}{\complement}(C)) \underset{\Phi}{\cap}(B \underset{\Phi}{\bigcap} \underset{\Phi}{\complement}(C)), \text { (by Theorems } 7 \& 6) \\
& \subseteq \quad(A \backslash C) \underset{\Phi}{\cap}(B \backslash C) \text {. (by Lemma } 7)
\end{aligned}
$$

Lemma 9. The descriptive difference of the descriptive intersection of two sets with one of these sets is the empty set.

$$
\begin{aligned}
& (A \underset{\Phi}{\cap} B) \backslash_{\Phi} A=\emptyset \\
& \left(A \bigcap_{\Phi} B\right) \backslash_{\Phi} B=\emptyset
\end{aligned}
$$

Proof.

$$
\begin{aligned}
\left(A \bigcap_{\Phi} B\right) \backslash_{\Phi} A & \subseteq(A \backslash A) \bigcap_{\Phi}(B \backslash A), \text { (by Lemma 8) } \\
& \subseteq \emptyset \bigcap_{\Phi}(B \backslash A),(\text { by Lemma } 2) \\
& =\emptyset .(\text { by Theorems } 6 \& 5) \\
(A \underset{\Phi}{\cap} B) \backslash_{\Phi} B & \left.\Longleftrightarrow\left(B \underset{\Phi}{\bigcap_{\Phi}} A\right) \backslash_{\Phi} B, \text { (by Theorem } 6\right) \\
& \Longleftrightarrow \emptyset .(\text { from above) }
\end{aligned}
$$

Lemma 10. Let $A, B$, and $C$ be sets. Then,

$$
(A \backslash \underset{\Phi}{\backslash} \underset{\Phi}{\cap} C=(A \underset{\Phi}{\cap} C) \underset{\Phi}{\backslash} B .
$$


Proof.

$$
\begin{aligned}
& x \in(A \backslash B) \underset{\Phi}{\cap} C, \\
\Longleftrightarrow & x \in A \cup C:(\Phi(x) \in \Phi(A) \text { and } \Phi(x) \notin \Phi(B)) \text { and } \Phi(x) \in \Phi(C), \text { (by Defns } 8 \& 9) \\
\Longleftrightarrow & x \in A \cup C: \Phi(x) \in \Phi(A) \text { and } \Phi(x) \notin \Phi(B) \text { and } \Phi(x) \in \Phi(C), \\
\Longleftrightarrow & x \in A \cup C:(\Phi(x) \in \Phi(A) \text { and } \Phi(x) \in \Phi(C)) \text { and } \Phi(x) \notin \Phi(B), \\
\Longleftrightarrow & \left.x \in(A \underset{\Phi}{\cap} C) \backslash_{\Phi} B \text {. (by Defns } 8 \& 9\right)
\end{aligned}
$$

Lemma 11. The descriptive intersection of the descriptive difference with the second set is the empty set.

$$
(A \backslash \underset{\Phi}{\backslash} B) \underset{\Phi}{\cap} B=\emptyset
$$

Proof.

$$
\begin{aligned}
(A \backslash \underset{\Phi}{ } B) \underset{\Phi}{\cap} B & \left.\Longleftrightarrow\left(A \bigcap_{\Phi} B\right) \backslash \underset{\Phi}{\backslash} B, \text { (by Lemma } 10\right) \\
& \Longleftrightarrow \emptyset .(\text { by Lemma } 9)
\end{aligned}
$$

Lemma 12. The descriptive intersection of a set and its relative descriptive complement of $B$ in $A$ is the empty set.

$$
B \underset{\Phi}{\cap} \complement_{\Phi}(B)=\emptyset
$$

Proof.

$$
\begin{aligned}
B \underset{\Phi}{\bigcap_{\Phi}} \complement_{A}(B) & \Longleftrightarrow(A \backslash B) \underset{\Phi}{\cap} B, \text { (by Theorem } 7 \text { \& Definition 10) } \\
& \Longleftrightarrow \emptyset .(\text { by Lemma } 11)
\end{aligned}
$$

Theorem 8. The descriptive intersection of a set and its descriptive complement is the empty set.

$$
A \underset{\Phi}{\cap} \underset{\Phi}{\complement}(A)=\emptyset
$$


Proof. Substitute $U$ for $A$ and $A$ for $B$ in Lemma 12.

Counter Example 3. The descriptive intersection of a set with the universe is not the original set.

$$
A \bigcap_{\Phi} U \neq A
$$

Proof. By Definition 8, $A \underset{\Phi}{\bigcap} U=\{x \in U: \Phi(x) \in \Phi(A)\}$. Let $x \in U \backslash A, a \in A$, and $\Phi(x)=\Phi(a)$. Then, $A \underset{\Phi}{\bigcap} U \neq A$.

Counter Example 4. The descriptive intersection of two sets is not a subset of each.

$$
\begin{aligned}
& A \underset{\Phi}{\cap} B \nsubseteq A \\
& A \underset{\Phi}{\cap} B \nsubseteq B
\end{aligned}
$$

Proof. By Definition 8.

Next, to prove Theorem 10, De Morgan's laws for set difference with respect to the descriptive approach presented here need to be given.

Theorem 9. Let $A, B, C$ be sets. Then,

$$
\begin{aligned}
& A \underset{\Phi}{\backslash}(B \underset{\Phi}{\cap} C)=(A \backslash B) \underset{\Phi}{\cup}(A \backslash C), \\
& A \backslash_{\Phi}(B \underset{\Phi}{\cup} C)=\left(A \backslash_{\Phi} B\right) \underset{\Phi}{\cap}(A \backslash C) .
\end{aligned}
$$

Proof.

$$
\begin{aligned}
x \in A \backslash \underset{\Phi}{ }(B \underset{\Phi}{\cap} C) & \Longleftrightarrow x \in A: \Phi(x) \notin \Phi(B \underset{\Phi}{\cap} C), \text { (by Definition 9) } \\
& \Longleftrightarrow x \in A: \Phi(x) \notin(\Phi(A) \cap \Phi(B)), \text { (by Definition 8) } \\
& \Longleftrightarrow x \in A: \Phi(x) \notin \Phi(B) \text { or } \Phi(x) \notin \Phi(C) \text {, (by De Morgan's Law) } \\
& \left.\Longleftrightarrow x \in\left(A \backslash \backslash_{\Phi} B\right) \underset{\Phi}{\cup}(A \backslash C) . \text { (by Definitions } 7 \& 9\right)
\end{aligned}
$$




$$
\begin{aligned}
& x \in A \backslash_{\Phi}(B \underset{\Phi}{\cup} C) \Longleftrightarrow x \in A: \Phi(x) \notin \Phi(B \underset{\Phi}{\cup} C) \text {, (by Definition 9) } \\
& \Longleftrightarrow \quad x \in A: \Phi(x) \notin(\Phi(A) \cup \Phi(B)) \text {, (by Definition 7) } \\
& \Longleftrightarrow \quad x \in A: \Phi(x) \notin \Phi(B) \text { and } \Phi(x) \notin \Phi(C) \text {, (by De Morgan's Law) } \\
& \Longleftrightarrow x \in(A \backslash B) \bigcap_{\Phi}(A \backslash C) \text {. (by Definitions } 8 \& 9 \text { ) }
\end{aligned}
$$

Theorem 10. Let $A, B$ be sets. Then,

$$
\begin{aligned}
& \complement_{\Phi}(A \underset{\Phi}{\cap} B)=\complement_{\Phi}(A) \underset{\Phi}{\cup} \complement_{\Phi}(B), \\
& \complement_{\Phi}(A \underset{\Phi}{\cup} B)=\complement_{\Phi}(A) \underset{\Phi}{\bigcap_{\Phi}}(B) .
\end{aligned}
$$

Proof.

$$
\begin{aligned}
& \complement_{\Phi}\left(A \bigcap_{\Phi} B\right) \Longleftrightarrow U \underset{\Phi}{\backslash}\left(A \bigcap_{\Phi} B\right), \text { (by Definition 11) } \\
& \Longleftrightarrow \quad\left(U \backslash \backslash_{\Phi} A\right) \underset{\Phi}{\cup}(U \backslash B), \text { (by Theorem 9) } \\
& \Longleftrightarrow \complement_{\Phi}(A) \underset{\Phi}{\cup} \complement_{\Phi}(B) \text {. (by Definition 11) } \\
& \complement_{\Phi}(A \cup \underset{\Phi}{\cup} B) \Longleftrightarrow U \underset{\Phi}{\backslash}\left(A \cup \cup_{\Phi} B\right), \text { (by Definition 11) } \\
& \Longleftrightarrow \quad(U \backslash A) \bigcap_{\Phi}(U \backslash B), \text { (by Theorem 9) } \\
& \Longleftrightarrow \quad \complement_{\Phi}(A) \bigcap_{\Phi} \underset{\Phi}{\complement}(B) \text {. (by Definition 11) }
\end{aligned}
$$

Counter Example 5. The descriptive intersection with subset is not the original subset.

$$
A \subseteq B \leftrightarrow A \bigcap_{\Phi} B=B
$$

Proof. Replace $U$ with $B$ in Counter Example 3.

Continuing on, Lemmas 13-16 are required to prove Corollary 1. Both Theorem 11 and its Corollary are in regard to the set theory property that compliments invert subsets. 
Lemma 13. The description of the descriptive complement of the descriptive complement of a set is the description of the set.

$$
\Phi\left(\underset{\Phi}{\complement}\left(\complement_{\Phi}(A)\right)\right)=\Phi(A)
$$

Proof.

$$
\begin{aligned}
\Phi(x) \in \Phi(\underset{\Phi}{\complement} \underset{\Phi}{(\complement}(A))) & \Longleftrightarrow \Phi(x) \in \Phi(U): \Phi(x) \notin \Phi\left(\complement_{\Phi}(A)\right), \text { (by Definition 11) } \\
& \Longleftrightarrow \Phi(x) \in \Phi(U): \Phi(x) \in \Phi(A), \text { (by Definition 11) } \\
& \Longleftrightarrow \Phi(x) \in \Phi(A) .
\end{aligned}
$$

Lemma 14. Descriptions of descriptive complements are equal if the descriptions of the original sets are equal.

$$
\left.\left.\Phi(A)=\Phi(B) \Longleftrightarrow \Phi \underset{\Phi}{\left(\complement_{\Phi}\right.}(A)\right)=\underset{\Phi}{\Phi} \underset{\complement}{(B)}\right)
$$

Proof.

$$
\begin{aligned}
\Phi(x) \in \Phi\left(\underset{\Phi}{\complement_{1}}(A)\right) & \Longleftrightarrow \Phi(x) \notin \Phi(A), \text { (by Definition 11) } \\
& \Longleftrightarrow \Phi(x) \notin \Phi(B), \quad(\text { since } \Phi(A)=\Phi(B)) \\
& \Longleftrightarrow \Phi(x) \in \Phi \underset{\Phi}{\left(\complement_{\Phi}(B)\right) .}
\end{aligned}
$$

Lemma 15. The description of the descriptive union of two sets is equal to the union of their descriptions.

$$
\Phi(A \cup \underset{\Phi}{\cup} B)=\Phi(A) \cup \Phi(B)
$$

Proof.

$$
\begin{aligned}
\Phi(x) \in \Phi(A \cup \underset{\Phi}{ } B) & \Longleftrightarrow \Phi(x) \in \Phi(A) \text { or } \Phi(x) \in \Phi(B), \text { (by Definition 7) } \\
& \Longleftrightarrow \Phi(A) \cup \Phi(B) .
\end{aligned}
$$

Lemma 16. The description of the descriptive intersection of two sets is equal to the intersection of their descriptions.

$$
\Phi(A \underset{\Phi}{\cap} B)=\Phi(A) \cap \Phi(B)
$$


Proof.

$$
\begin{aligned}
\Phi(x) \in \Phi(A \underset{\Phi}{\cap} B) & \Longleftrightarrow \Phi(x) \in \Phi(A) \text { and } \Phi(x) \in \Phi(B), \text { (by Definition 8) } \\
& \Longleftrightarrow \Phi(A) \cap \Phi(B) .
\end{aligned}
$$

Theorem 11. The descriptive complement inverts subsets.

$$
A \subseteq B \Longleftrightarrow \complement_{\Phi}(B) \subseteq \complement_{\Phi}(A)
$$

Proof.

$$
\begin{aligned}
x \in \complement_{\Phi}(B) & \Longleftrightarrow x \in U \text { and } \Phi(x) \notin \Phi(B), \text { (by Definition } 11) \\
& \Longleftrightarrow x \notin B,(\text { by Definition } 6) \\
& \Longleftrightarrow x \notin A,(\text { since } A \subseteq B) \\
& \Longleftrightarrow x \in \underset{\Phi}{\complement}(A) .(\text { by Definition } 11)
\end{aligned}
$$

Further,

$$
\begin{aligned}
x \in \complement_{\Phi}(A) & \Longleftrightarrow x \in U \text { and } \Phi(x) \notin \Phi(A), \text { (by Definition 11) } \\
& \Longleftrightarrow x \notin A, \text { (by Definition } 6) \\
& \Longleftrightarrow x \in B \backslash A \text { or } x \in U \backslash B,(\text { since } A \subseteq B)
\end{aligned}
$$

where,

$$
\begin{aligned}
x \in B \backslash A & \Longleftrightarrow x \in B \text { and } x \notin A, \\
& \Longleftrightarrow \Phi(x) \in \Phi(B) \text { and } \Phi(x) \notin \Phi(A), \text { (by Definition } 6) \\
& \Longleftrightarrow x \notin \underset{\Phi}{\complement}(B),(\text { by Definition } 11)
\end{aligned}
$$

and

$$
x \in U \backslash B \quad \Longleftrightarrow \quad x \in \underset{\Phi}{\complement}(B) \text { or } x \notin \underset{\Phi}{\complement}(B) \text {. (by Lemma 5) }
$$

Consequently, $x \in \underset{\Phi}{\complement}(B)$ implies that $x \in \underset{\Phi}{\complement}(A)$, but $x \in \underset{\Phi}{\complement}(A)$ implies that $x$ may or may not be in $\underset{\Phi}{\complement}(B)$. Thus, $A \subseteq B \Longleftrightarrow \underset{\Phi}{\complement}(B) \subseteq \underset{\Phi}{\complement_{\Phi}}(A)$. 
Corollary 1. The descriptive complement inverts description subsets.

$$
\Phi(A) \subseteq \Phi(B) \Longleftrightarrow \Phi(\underset{\Phi}{\complement}(B)) \subseteq \underset{\Phi}{\left(\complement_{\Phi}(A)\right)}
$$

Proof.

$$
\begin{aligned}
& \Phi(A) \subseteq \Phi(B) \\
& \Longleftrightarrow \Phi(A) \cap \Phi(B)=\Phi(A) \text {, (intersection with subset is subset) } \\
& \Longleftrightarrow \Phi\left(A \underset{\Phi}{\bigcap_{\Phi}} B\right)=\Phi(A), \text { (by Lemma 16) } \\
& \Longleftrightarrow \Phi\left(\complement_{\Phi}\left(A \bigcap_{\Phi} B\right)\right)=\Phi(\underset{\Phi}{\complement}(A)), \text { (by Lemma 14) } \\
& \left.\Longleftrightarrow \Phi(\underset{\Phi}{\complement}(A) \underset{\Phi}{\cup} \underset{\Phi}{\complement}(B))=\Phi \underset{\Phi}{\left(\complement_{\Phi}\right.}(A)\right) \text {, (by Theorem 10) } \\
& \Longleftrightarrow \Phi\left(\complement_{\Phi}(A)\right) \cup \Phi\left(\underset{\Phi}{\complement_{\Phi}}(B)\right)=\Phi(\underset{\Phi}{\complement}(A)) \text {, (by Lemma 15) } \\
& \Longleftrightarrow \complement_{\Phi}(B) \subseteq \complement_{\Phi}(A) \text {. (union with superset is superset) }
\end{aligned}
$$

Theorem 12. The descriptive intersection is empty if one of the sets is the subset of the descriptive complement.

$$
A \bigcap_{\Phi} B=\emptyset \Longleftrightarrow A \subseteq \underset{\Phi}{\complement}(B)
$$

Proof.

$$
\begin{aligned}
A \underset{\Phi}{\cap} B=\emptyset & \Longleftrightarrow \forall x \in A \cup B, \Phi(x) \notin \Phi(A) \text { or } \Phi(x) \notin \Phi(B), \text { (by Definition 8) } \\
& \Longleftrightarrow \forall x \in A, \Phi(x) \notin \Phi(B), \text { (by Definition } 6) \\
& \Longleftrightarrow A \subseteq \complement_{\Phi}(B) \text { (by Definition 11). }
\end{aligned}
$$

Similarly for $B$.

Theorem 13. A union between two sets that produces the universe implies the descriptive complement of one of the sets is the subset of the other.

$$
A \cup B=U \Longleftrightarrow \complement_{\Phi}(A) \subseteq B
$$


Proof.

$$
\begin{aligned}
x \in \complement_{\Phi}(A) & \Longleftrightarrow x \in U: \Phi(x) \notin \Phi(A), \text { (by Definition 11) } \\
& \Longleftrightarrow x \in U \backslash A: \Phi(x) \notin \Phi(A), \\
& \Longleftrightarrow x \in B: \Phi(x) \notin \Phi(A), \text { (since } A \cup B=U) \\
\Longrightarrow A \cup B=U & \Longleftrightarrow \underset{\Phi}{\prod_{\Phi}(A) \subseteq B .}
\end{aligned}
$$

Counter Example 6. Set union does not distribute over descriptive intersection.

$$
A \cup(B \underset{\Phi}{\cap} C) \neq(A \cup B) \bigcap_{\Phi}(A \cup C)
$$

Proof. Let $a_{1}, a_{2} \in A, b \in B, c \in C, c \notin A: \Phi\left(a_{1}\right)=\Phi(b), \Phi\left(a_{2}\right)=\Phi(c), \Phi\left(a_{1}\right) \notin \Phi(C)$, and $\Phi\left(a_{2}\right) \notin \Phi(B)$. Then, $b, c \notin B \bigcap_{\Phi} C$, and, consequently, $b, c \notin A \cup\left(B \bigcap_{\Phi} C\right)$. However, $a_{1}, a_{2}, b \in A \cup B, a_{1}, a_{2}, c \in A \cup C$, and, as a result, $b, c \in(A \cup B) \underset{\Phi}{\bigcap}(A \cup C)$.

Theorem 14. Descriptive intersection is distributive over set union.

$$
A \bigcap_{\Phi}(B \cup C)=\left(A \bigcap_{\Phi} B\right) \cup\left(A \bigcap_{\Phi} C\right)
$$

Proof.

$$
\begin{aligned}
& x \in A \bigcap_{\Phi}(B \cup C), \\
\Longleftrightarrow & x \in A \cup B \cup C: \Phi(x) \in \Phi(A) \text { and, either, } \Phi(x) \in \Phi(B) \text { or } \Phi(x) \in \Phi(C), \\
\Longleftrightarrow & x \in A \cup B \cup C:(\Phi(x) \in \Phi(A) \text { and } \Phi(x) \in \Phi(B)) \text { or }(\Phi(x) \in \Phi(A) \text { and } \Phi(x) \in \Phi(C)), \\
\Longleftrightarrow & x \in\left(A \bigcap_{\Phi} B\right) \cup\left(A \bigcap_{\Phi} C\right) .
\end{aligned}
$$

\section{Application to Description-based Neighbourhoods}

Section 3 introduced descriptive operators and their properties, but made no stipulation about how the set operands are formed. This section outlines several types of neighbourhoods to which the above operators can be applied.

Definition 12. Spatial Neighbourhood (without focus). A spatial neighbourhood without focus is a traditionally defined set, i.e. it is a collection of objects. 
The set operands from Examples 1-4 are examples of spatial neighbourhoods without focus, which are simply collections of pixels.

Definition 13. Spatial Neighbourhood (with focus). Let $x, y \in O$ be perceptual objects, let $d(x, y)$ denote any form of distance metric between $x$ and $y$, and let $N_{d}(x, \mathfrak{r})=\{y \in O: d(x, y)<\mathfrak{r}\}$ denote an open ball (using any distance metric $d(x, y)$ ) with with radius $\mathfrak{r} \in[0, \infty)$, and centre $x$. Then, a spatial neighbourhood with focus $x$ is defined as $N_{d}(x, \mathfrak{r})$ for some $x \in O$.

The term focus used here is synonymous with the centre associated with an open ball, yet is preferred (in this context) since spatial neighbourhoods may still have an object that can be considered the spatial centre of the set. Moreover, the term focus implies conscious directed attention, which is more in line with the idea of using description-based neighbourhoods as part of a formal framework for quantifying the perceptual nearness of objects and sets of objects.

Definition 14. Description-Based Neighbourhood. Let $x, y \in O$ be perceptual objects with object descriptions given by $\Phi(x), \Phi(y)$, and let $\varepsilon \in \mathbb{R}$. Then, a description-based neighbourhood is defined as

$$
\mathfrak{N}_{x, \varepsilon}=\{y \in O:|\Phi(x)-\Phi(y)|<\varepsilon\} .
$$

A point $y$ is a member of $\mathfrak{N}_{x, \varepsilon}$, if and only if, $|\Phi(x)-\Phi(y)|<\varepsilon$.

Example 6. Consider a perceptual system defined in a manner similar to Example 1. Then, the inverted pixels in Fig. 8b represent a description-based neighbourhood, where the focus (centre) of the neighbourhood is represented by the enlarged dark pixel. Note, $\varepsilon=0.23$ (out of a maximum of $\sqrt{3}$ ) was used to generate this neighbourhood.

Definition 15. Bounded-Descriptive Neighbourhood. Let $x, y \in O$ be perceptual objects with object descriptions given by $\Phi(x), \Phi(y)$, and let $\varepsilon \in \mathbb{R}$. Then, a bounded-descriptive neighbourhood is defined as

$$
\mathfrak{N}_{x, \varepsilon}^{\circ}=\left\{y \in O:|\Phi(x)-\Phi(y)<\varepsilon| \text { and } y \in N_{d}(x, \mathfrak{r})\right\} .
$$

In other words, a point $y$ is a member of $\mathfrak{N}_{x, \varepsilon}^{\circ}$, if and only if, $y$ is descriptively similar to some point $z$ inside $N_{d}(x, \mathfrak{r})$ with centre $x$ and radius $\mathfrak{r}$.

Example 7. As in all the previous examples, assume a perceptual system similar to Example 1. Then, the inverted pixels in Fig. 8d represent a bounded-descriptive neighbourhood, where the focus (centre) of the neighbourhood is the enlarged green pixel. Here, $\varepsilon=0.17$ was used to generate this neighbourhood. 


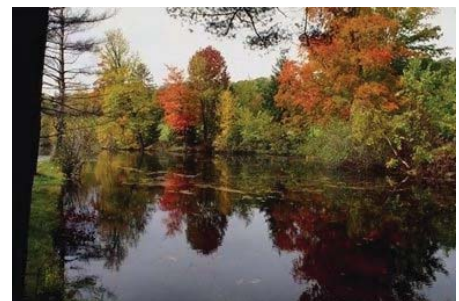

(a)

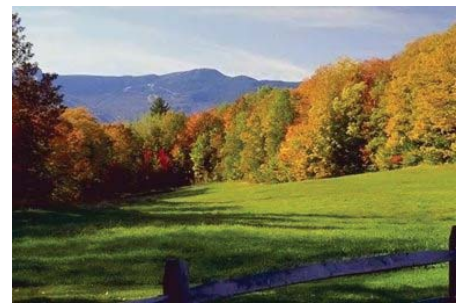

(c)

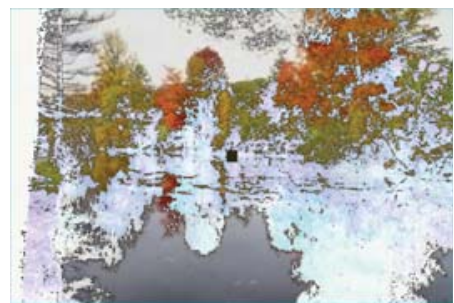

(b)

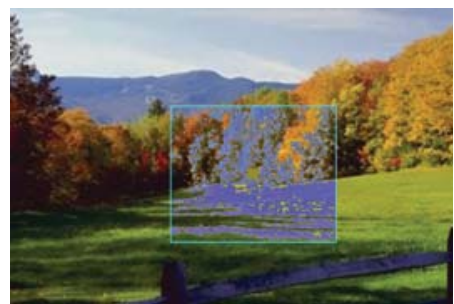

(d)

Figure 8. Example demonstrating Definitions $13 \& 14$.

\section{Metric-Free Nearness Measure}

This section introduces a metric-free description-based nearness measure using the descriptive operators introduced in Section 3, and is related to work on a tolerance-based nearness measure reported in $[16,17]$. Furthermore, the approach presented here has direct application to image analysis and is related to the rough set image analysis approaches reported in $[25,26,27,28,29,30,31,32]$. Similarly, this measure can be applied to the problem of content-based image retrieval [33] in a manner similar to the tolerance nearness measure approached taken in $[34,35,36]$. As in the case of the tolerance nearness measure, both approaches aim to quantify the similarity between sets of objects based on object description. However, the tolerance nearness measure is obtained using tolerance classes (see, e.g. [37]) obtained from the union of the sets under consideration, while the description-based nearness measure is based on the descriptive operators presented in this article. The idea that motivated this measure comes from the observation in [1] that nearness is considered a generalization of intersection. Intuitively speaking, we perceive sets of objects to be similar or near in some manner when they share common characteristics. Thus, if considering set descriptions (as given in Definition 6), the descriptive intersection should not be empty if we consider the sets to be similar with respect 
TABLE 2. Nearness Measure Values for Images in Fig. 9

\begin{tabular}{ccc}
\hline Image & Neighbourhood Type & $d N M$ \\
\hline Fig. 9a & Spatial (without focus) & 0.54 \\
Fig. 9b & & 0.74 \\
Fig. 9d & Bounded-Descriptive Neighbourhood & 0.37 \\
Fig. 9f & 1 \\
\hline
\end{tabular}

to one or more features. Keeping these ideas in mind, a metric-free description-based nearness measure, $d N M$, is defined as follows.

Definition 16. Metric-Free Description-Based Nearness Measure. Let $X, Y \subseteq O$ be sets of perceptual objects within a perceptual system. Then, a metric-free description-based nearness measure is defined as

$$
d N M(X, Y)=1-\frac{|X \underset{\Phi}{\cap} Y|}{|X \cup Y|} .
$$

The nearness measure produces values in the interval [0,1], where, for a pair of sets $X, Y, a$ value of 0 represents complete resemblance, and a value of 1 intimates no resemblance.

Example 8. Consider a perceptual system defined in a manner similar to Example 1, except using only probe functions based on the red and green components from the RGB colour model. Then, the $d N M$ values of the images in Fig. 9 are given in Table 2, where two different types of neighbourhoods are considered in the descriptive intersection. Specifically, Fig. 9a \& 9b contain spatial neighbourhoods, and Fig. 9c \& 9e depict the bounded-descriptive neighbourhoods (obtained with $\varepsilon=0.23$ ) that are used in generating the descriptive intersection given in Fig. 9d \& 9f. Notice, as expected, in all cases the $d N M$ is lower when comparing the two mushrooms. Also, there are no objects in the two neighbourhoods in Fig. 9e that have matching descriptions. Hence, the empty intersection and $d N M=1$.

\section{Conclusion}

This article presented several new description-based operators and some of their properties, and a new metric-free description-based nearness measure. The Proximity System was used to demonstrate practical application of these description-based operators to digital images. Moreover, the results presented in Section 5 lay the foundation for applying the $d N M$ introduced here to the problem of content-based image retrieval. Future work will consist of the application 


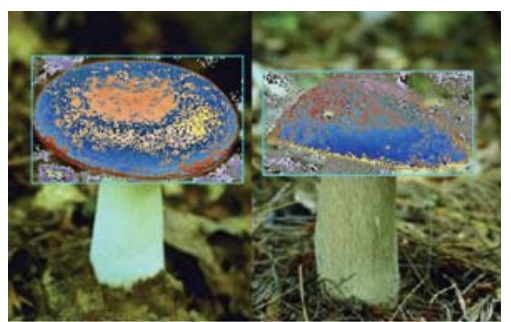

(a)

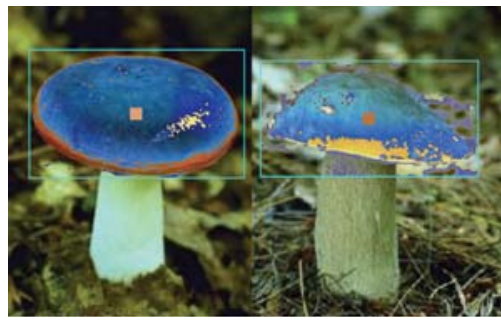

(c)

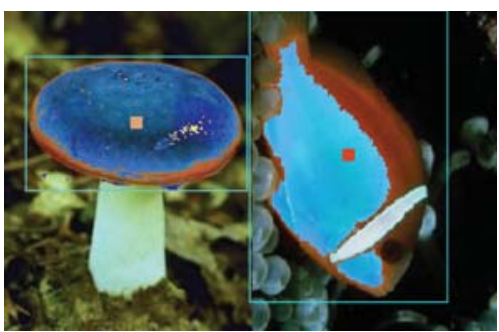

(e)

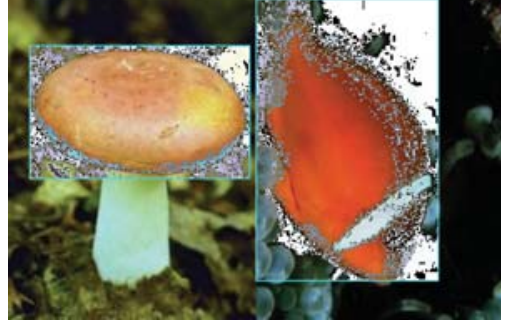

(b)

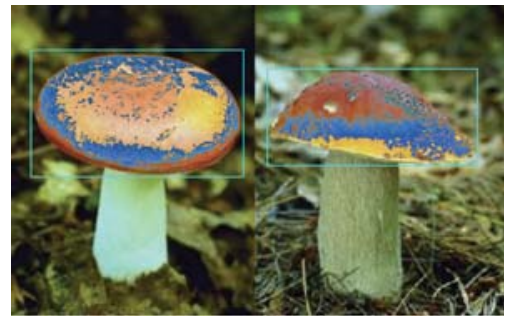

(d)

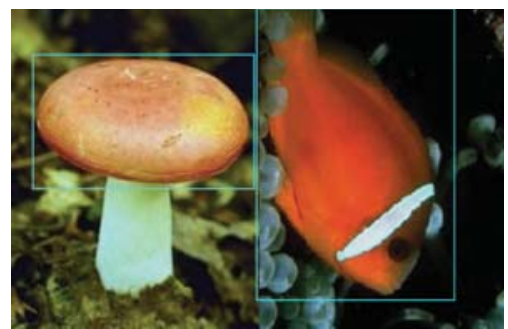

(f)

Figure 9. Example demonstrating Definition 16.

of the $d N M$ to content-based image retrieval problems and comparison of the $d N M$ with the established $t N M$.

\section{Acknowledgements}

The author extends his profound thanks to James F. Peters for many helpful discussions and important suggestions that inspired the ideas presented in this paper. Also, many thanks to Garrett Smith for writing the code for the Proximity System. 


\section{References}

[1] Peters, J.F., Naimpally, S.A.: Applications of near sets. Notices of the American Mathematical Society 59(4) (2012) 536-542

[2] Naimpally, S.A.: Near and far. A centennial tribute to Frigyes Riesz. Siberian Electronic Mathematical Reports 6 (2009) A.1-A.10

[3] Naimpally, S.A., Peters, J.F.: Topology with Applications.Topological Spaces via Near and Far. World Scientific, Singapore (2013) In Press.

[4] Di Concilio, A.: Proximity: A powerful tool in extension theory, function spaces, hyperspaces, boolean algebras and point-free geometry. In Mynard, F., Pearl, E., eds.: Beyond Topology. American Mathematical Society (2009)

[5] Sossinsky, A.B.: Tolerance space theory and some applications. Acta Applicandae Mathematicae: An International Survey Journal on Applying Mathematics and Mathematical Applications 5(2) (1986) 137-167

[6] Poincaré, H.: Science and Hypothesis. The Mead Project, Brock University (1905) L. G. Ward's translation.

[7] Benjamin, Jr., L.T.: A Brief History of Modern Psychology. Blackwell Publishing, Malden, MA (2007)

[8] Hergenhahn, B.R.: An Introduction to the History of Psychology. Wadsworth Publishing, Belmont, CA (2009)

[9] Zeeman, E.C.: The topology of the brain and the visual perception. In Fort, K.M., ed.: Topoloy of 3-manifolds and selected topices. Prentice Hall, New Jersey (1965) 240-256

[10] Naimpally, S.A., Warrack, B.D.: Proximity spaces. In: Cambridge Tract in Mathematics No. 59. Cambridge University Press, Cambridge, UK (1970)

[11] Pawlak, Z., Peters, J.F.: Jak blisko (how near). Systemy Wspomagania Decyzji I (2002) 57, 109.

[12] Peters, J.F.: Near sets. General theory about nearness of objects. Applied Mathematical Sciences 1(53) (2007) 2609-2629

[13] Peters, J.F.: Near sets. Special theory about nearness of objects. Fundamenta Informaticae 75(1-4) (2007) 407-433

[14] Peters, J.F.: Tolerance near sets and image correspondence. International Journal of Bio-Inspired Computation 1(4) (2009) 239-245

[15] Peters, J.F.: Corrigenda and addenda: Tolerance near sets and image correspondence. International Journal of Bio-Inspired Computation 2(5) (2010) 310-318

[16] Henry, C.J.: Near Sets: Theory and Applications. PhD thesis (2010) Available at: https://mspace. lib.umanitoba.ca/handle/1993/4267. 
[17] Henry, C.J.: Perceptually indiscernibility, rough sets, descriptively near sets, and image analysis. Transactions on Rough Sets LNCS 7255 (2012) 41-121

[18] Henry, C.: Near set Evaluation And Recognition (NEAR) system. In Pal, S.K., Peters, J.F., eds.: Rough Fuzzy Analysis Foundations and Applications. CRC Press, Taylor \& Francis Group (2010) 7-1 - 7-22 exe. availabe at: http://wren.ee.umanitoba.ca.

[19] Pavel, M.: Fundamentals of Pattern Recognition. Marcel Dekker, Inc., NY (1993)

[20] Peters, J.F.: Classification of objects by means of features. In: Proceedings of the IEEE Symposium Series on Foundations of Computational Intelligence (IEEE SCCI 2007). (2007) 1-8

[21] Peters, J.F.: Classification of perceptual objects by means of features. International Journal of Information Technology \& Intelligent Computing 3(2) (2008) 1 - 35

[22] Peters, J.F., Wasilewski, P.: Foundations of near sets. Info. Sci. 179(18) (2009) 3091-3109

[23] Duda, R., Hart, P., Stork, D.: Pattern Classification. 2nd edn. Wiley (2001)

[24] Bourbaki, N.: Elements of Mathematics: Theory of Sets. Hermann, Publishers in Arts and Science, Paris (1968)

[25] Pal, S.K., Mitra, P.: Multispectral image segmentation using rough set initialized em algorithm. IEEE Transactions on Geoscience and Remote Sensing 11 (2002) 24952501

[26] Peters, J.F., Borkowski, M.: k-means indiscernibility over pixels (2004)

[27] Pal, S.K., Shankar, B.U., Mitra, P.: Granular computing, rough entropy and object extraction. Pattern Recognition Letters 26(16) (2005) 401-416

[28] Borkowski, M., Peters, J.F.: Matching 2d image segments with genetic algorithms and approximations spaces. Transactions on Rough Sets LNCS 4100 (2006)

[29] Borkowski, M.: 2D to 3D Conversion with Direct Geometrical Search and Approximation Spaces. PhD thesis (2007)

[30] Maji, P., Pal, S.K.: Maximum class separability for rough-fuzzy c-means based brain mr image segmentation. Transactions on Rough Sets IX, LNCS-5390 (2008) 114-134

[31] Mushrif, M., Ray, A.K.: Color image segmentation: Rough-set theoretic approach. Pattern Recognition Letters 29(4) (2008) 483-493

[32] Hassanien, A.E., Abraham, A., Peters, J.F., Schaefer, G., Henry, C.: Rough sets and near sets in medical imaging: A review. IEEE Transactions on Information Technology in Biomedicine 13(6) (2009) 955-968

[33] Smeulders, A.W.M., Worring, M., Santini, S., Gupta, A., Jain, R.: Content-based image retrieval at the end of the early years. IEEE Transactions on Pattern Analysis and Machine Intelligence 22(12) (2000) 1349-1380 
[34] Henry, C.J., Ramanna, S., Levy, D.: Quantifying nearness in visual spaces. Cybernetics and Systems Journal (2012) under review.

[35] Henry, C.J., Ramanna, S.: Maximal clique enumeration in finding near neighbourhoods. Transactions on Rough Sets (2012) under review.

[36] Henry, C.J., Ramanna, S.: Signature-based perceptual nearness. application of near sets to image retrieval. Mathematics in Computer Science (2012) under review.

[37] Peters, J.F., Wasilewski, P.: Tolerance spaces: Origins, theoretical aspects and applications. Information Sciences 195(0) (2012) 211-225

Christopher J. Henry

Department of Applied Computer Science, University of Winnipeg, Canada, R3B 2E9

e-mail: ch.henry@uwinnipeg.ca 